\title{
True temperature measurement of electronics through infrared transparent materials
}

\author{
by R. Lehtiniemi, C.-M. Fager and J. Rantala
}

Nokia Research Center, P.O. Box 407, FIN-00045 NOKIA GROUP, Finland

\begin{abstract}
The applications of thermography in electronics have increased considerably during the last years, simultaneously with more and more emerging thermal design problems. However, most often the infrared cameras have been utilized only for verification of thermal design of already built printed circuit boards in free air, which seldom is their natural environment of use. In this work, possibility to perform true temperature measurements of electronics located inside an enclosure with assist of infrared transparent materials is studied.
\end{abstract}

\section{Introduction}

Recently, the thermal management in electronics has become more and more essential because of rapidly increasing power densities at all assembly levels. An example of this is depicted in Fig. 1. The complete mathematical analysis of the phenomena related with thermal management, especially the solution of such governing partial differential equations as the Navier-Stokes equations and the energy conservation equation, can often be extremely difficult if possible at all. However, experience together with simplifications and numerical methods can be utilized to enable a reasonable thermal design process.

Because of the uncertainty of validity of assumptions made, the design has to be verified by measurements. Since the majority of the surfaces to be measured, such as printed circuit boards (PCB), are far from isothermal, traditional point temperature measurements for example with thermocouples seldom provide enough information for making the final decisions about the quality of thermal design. One of the available techniques, which may considerably facilitate this evaluation process, is infrared (IR) thermography. With an IR camera, it is often straightforward and fast to verify the success of the thermal design either by capturing single thermal snapshot images or by recording image sequences of transient phenomena like cold start.

The standard use of thermal imaging in electronics applications today has been the search for hot spots in powered PCBs located in free air. However, almost always in reality the target is located inside of a chassis or a subrack. If the object has been removed from its intended operating environment, also the conditions are changed even drastically from those, which predominate during the real use. Thus, the thermal data obtained this way does not contain information about the true temperatures or the correct temperature distribution during the actual operation. Worst of all, the real operating temperatures tend to be underestimated, especially with devices cooled by natural convection.

Unfortunately, not very often the actual electronic object can be imaged in its real environment of use. Usually at least one wall of the enclosure blocks the direct optical path from camera to target, which impedes standard thermal imaging. One possible procedure to avoid this problem is to switch off the electronic device after it has gained thermal equilibrium in its normal usage environment, remove it from the enclosure, sample its thermal behaviour during the cooling phase, and finally extrapolate the cooling curves backwards in time to predict the actual temperatures in the real use conditions. Nevertheless, also this method includes inaccuracies and may thus sometimes be proven inadequate.

In our ongoing work, the possibility to perform reliable and reproducible true temperature measurements of electronic devices located inside an enclosure is studied. For this purpose, infrared transparent materials have been and will be utilized as replacements of wall of wind 
tunnels and device enclosures. Thus the direct viewing of the target under the real conditions has been enabled. However, the introduced extra medium in the optical path always causes apparent decrease in thermographically measured temperature. As an example of this effect and its compensation by a calibration procedure, a simple series of measurements is described in the following.

\section{Demonstrative experimental set-up and measurements}

The device used in the following measurement demonstration was a standard thermal test die mounted in a 208-lead Plastic Quad Flat Package (PQFP-208). The test die consists essentially of a resistive heater and a $p$ - $n$ junction. The heating power is fed to the resistor, and the resulted junction temperature increment is measured directly by the thermal behaviour of the current/voltage characteristic curve of the $p-n$ junction. The PQFP component was surface mounted onto a standard single component test PCB, defined by a JEDEC standard [1]. Both the component and the board were sprayed with a thin layer of matt black paint with a known, measured emissivity of 0.92 .

The test PCB was mounted in a vertical position in a wind tunnel test chamber (Fig. 2.) so that the direction of the airflow was against gravity. One wall of the chamber had an aperture for direct viewing of the test PQFP. The case temperature measurements were

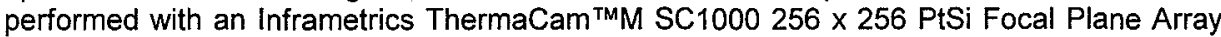
(FPA) infrared imaging system [2] operating in the 3.4 to $5 \mu \mathrm{m}$ spectral range. A 16 degree, $1: 1$ view standard lens with diffractive optics was applied. For comparison, also a corresponding $320 \times 240$ pixels Agema 550 FPA camera was used [3].

In the measurements, the wind tunnel was used both as a still-air chamber and as an actual wind tunnel with $2 \mathrm{~m} / \mathrm{s}$ air velocity. The test chip was heated with two different power levels, $1.5 \mathrm{~W}$ and $3.5 \mathrm{~W}$, for both air speeds. In all four cases, the thermographic case temperature measurement through the wall orifice was performed in two ways: first through the open aperture, and second by covering the window with a thin polymeric film.

When the IR camera in use allows the user to enter an additional transmittance value for the external optics, it is easy to calibrate the camera with a known temperature value to find the apparent transmittance. However, if this option is not available, the built-in atmospheric transmission model can be utilized in the same manner. In this somewhat crude but simple technique the imaging distance can be used as the calibration parameter: Instead of varying the input for the external transmittance until the correct measurement result has been obtained, the distance can be altered maintaining the fixed value of relative humidity. Both these above-mentioned techniques were utilized to study the effect of the film on the measured temperature and its compensation.

The plastic film utilized in these measurements was 76 micrometers thick, octene-based low-density polyethylene (LDPE) DuPont SM-3 Sclairfilm\&. Sclairfilm $\otimes$ is a sealant film that combines strength and flex-crack characteristics with heat seal and hot tack properties, being thus quite a suitable film for IR window purposes. Because of its about three times higher than normal puncture, impact, and tear strength, Sclairfilm $\otimes$ film allows up to $50 \%$ downgauging of the sealant layer in industrial applications. The infrared transmittance spectrum of Sclairfilm $\otimes$ is known from the IR spectroscopic measurements provided by the manufacturer (Fig. 3a). For comparison, also standard domestic LDPE film was tested and its transmittance was measured (Fig. 3b). Only the transmittance values for the short wavelength range are depicted here.

In addition to thermographic temperature measurements, reference values for the case temperature were recorded with two calibrated high grade K-type thermocouples. The thermocouples were attached on the component case with Kapton tape. One was located on the middle of the component case right on top of the die and the other on the corner of the component in order to monitor the temperature distribution over the component surface as a reference for the IR measurement. During the thermographic temperature registration, the thermocouple over the die was removed so that it would not interfere with the measurement. 


\section{Examples of results and discussion}

A typical thermal image captured during the measurements is shown in Fig. 4. Both the test board and the test component are visible in the image. Both the location and the size of the die distinguish clearly as the warmest area in the middle of the component. Also the thermocouple wire attached on the left side of the component can be seen.

A simple example of the results with external transmission variation is presented in Fig. 5. The measurements depicted here were performed in nearly still-air conditions with the heating power of $1.5 \mathrm{~W}$. In all different cases, represented by the four columns of the graph (Case\#), three different temperatures are plotted with their error bars $\left( \pm 2^{\circ} \mathrm{C}\right.$ for the IR measurement and $\pm 0,2^{\circ} \mathrm{C}$ for the thermocouple and for the $\mathrm{p}-\mathrm{n}$ measurement). The junction temperature, $\mathrm{Tj}$, was measured with the $\mathrm{p}-\mathrm{n}$ junction, the case temperature, $\mathrm{Tc}, \mathrm{TC}$, with a thermocouple directly over the die area, and the thermographic temperature, TIR, with the ThermaCamTM IR camera.

In the first column of the graph (Case 1), the temperature values for the case where the orifice on the wind tunnel wall was open are plotted. As can be seen, the measurements are relatively congruent, although the IR measurement tends to slightly exaggerate the case temperature. In Case 2, the orifice is covered with a layer of Sclairfilm®. The IR temperature drops $5.0^{\circ} \mathrm{C}$, as expected when the influence of the film is not compensated with external transmittance correction. The correct input value for the external transmittance can in some extent be estimated from the measurements or manufacturer data such as graphs presented in Figs. 3 a) and b). In Case 3, an estimate of $75 \%$ transmission is used. Correction seems to be quite right. However, it is easy to overcompensate the transmittance: In the last Case 4 the transmittance is $60 \%$ and the IR measurement overestimates the temperature by $5.0^{\circ} \mathrm{C}$. $n$ other measurements, the transmittance of $75 \%$ gave case temperature values devia1ting less than $\pm 1.3^{\circ} \mathrm{C}$ from the thermocouple measurement.

In Fig. 6, results of a similar measurement are shown. In this example, the effect of the film was compensated by using the camera's built-in atmospheric attenuation model. Varying the nominal measurement distance it is possible to find the thickness of the air layer in the model, which corresponds to the attenuation of the film. As can be seen in the distance variation sequence in the graph, the distance of $40 \mathrm{~m}$ (precisely $43 \mathrm{~m}$ ) produces a correct rectification.

As summary, it is possible to compensate the effect of an additional IR transparent material by using the built-in features of modern IR cameras with reasonable amount of calibration work. The simple measurements performed here suggest that the calibration at a single value of temperature and air flow velocity provides adequate accuracy from the point of view of electronics thermal management, but that the more doubtful distance correction has to be done separately for each set of parameters. However, the subject will be studied in more details in near future; for instance possibility to utilize actual solid IR window materials in practical electronics is rather interesting.

\section{Acknowledgements}

The authors would like to thank Dr. John Lohan and Mr. Peter Rodgers from Nokia Research Center for their invaluable advice and Ms. Terhi Vikki-Pere for performing the IR transmittance measurements. Also Mr. Esko Virtanen from Inframetrics OY, Mr. C.A.I. Kabrell from Nokia Telecommunications/Professional Mobile Radio, and Mr. Mario Lutterotti from DuPont Canada Inc. are thanked for their support.

\section{REFERENCES}

[1] EIA/JESD 51-3. - Low effective thermal conductivity test board for leaded surface mount packages. Electronics Industries Association, August 1996.

[2] http://www.inframetrics.com/products/tcam/sc1000/index.htm

[3] http://www.flir.com/products/cm/550.htm 
http://dx.doi.org/10.21611/qirt.1998.028

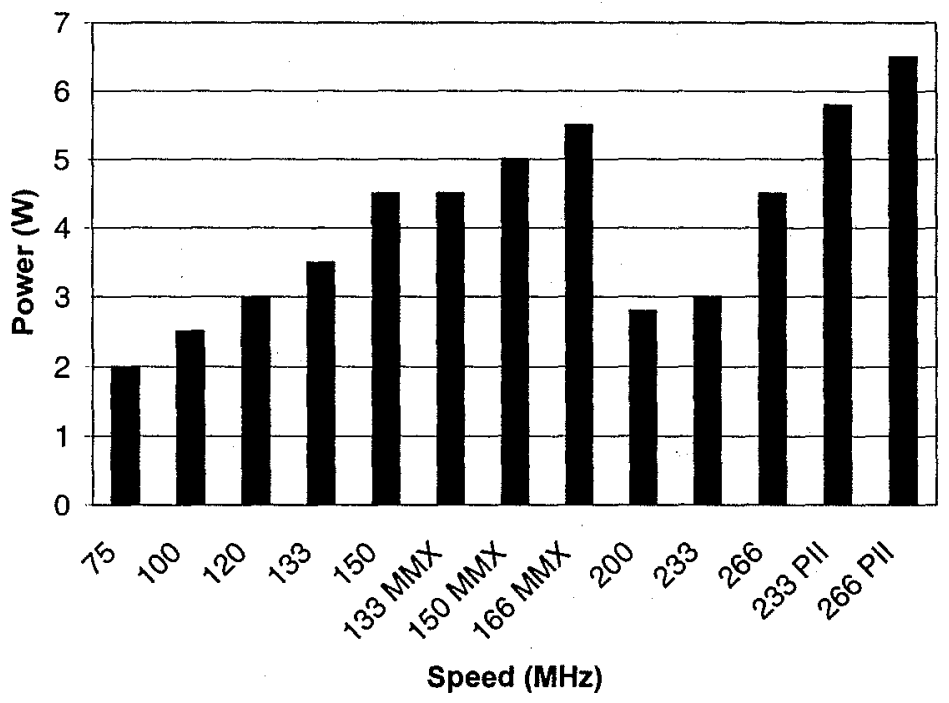

Fig. 1. Resent development of active power dissipation levels for mobile PC processors, the temporary power reduction after the MMX processor is due to the new $0.25 \mu \mathrm{m}$ technology
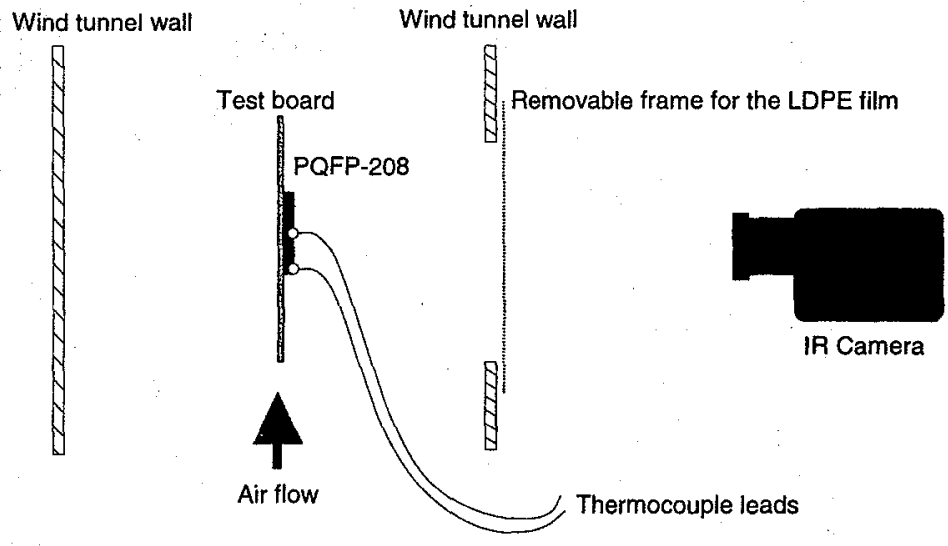

Fig. 2. Setup of the experimental setup 
http://dx.doi.org/10.21611/qirt.1998.028
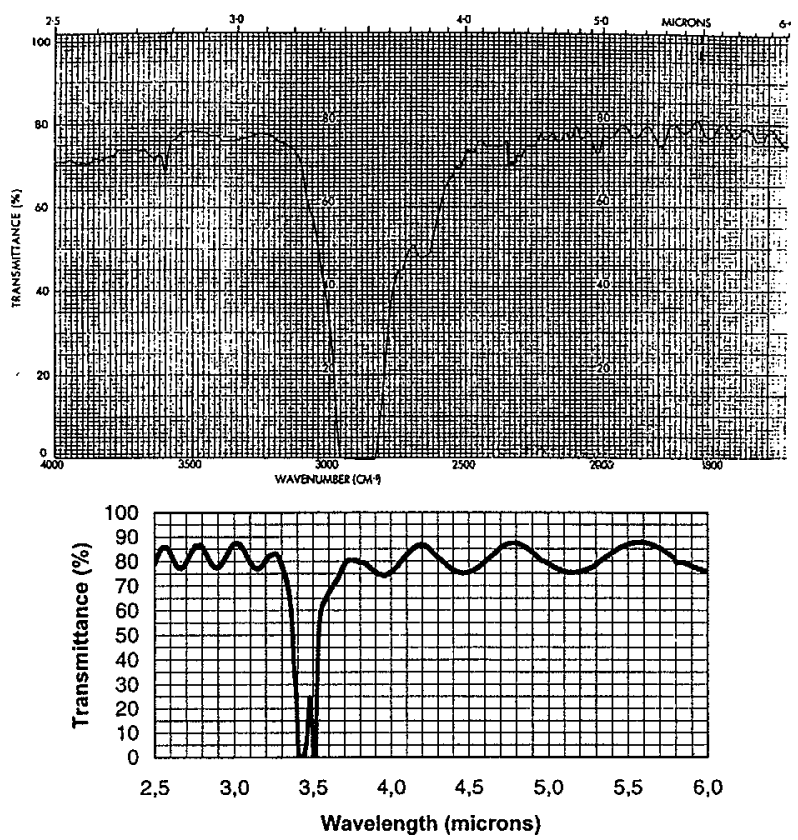

Fig. 3. Transmittance spectra of a) DuPoint Sclairfilm and b) standard domestic LDPE film

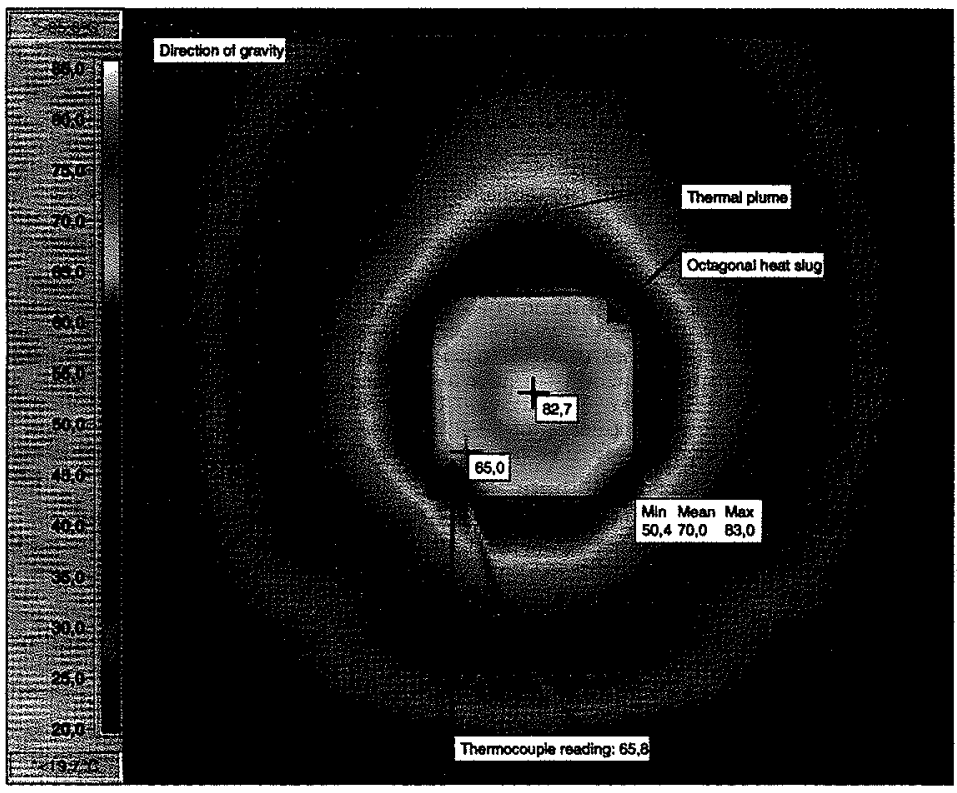

Fig. 4. A typical Irimage during measurements 
http://dx.doi.org/10.21611/qirt.1998.028

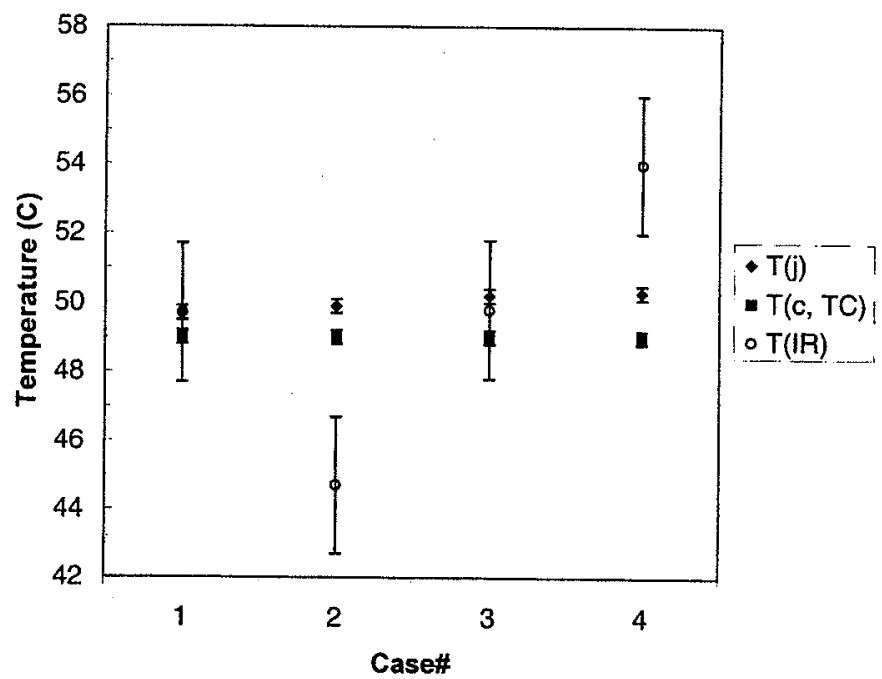

Fig. 5. An example of the measurent results. The junction temperature $T(j)$ is measured with an internal $\mathrm{p}$-n junction and the case temperature is measured both with thermocuple, T(c,TC, and with IR camera T(IR), in four cases: Case 1 with direct view of the sample, Case 2 through a LDPE film, Case 3 also through film but with an external transmittance of $75 \%$, and Case 4 throuh film an external transmittance of $60 \%$

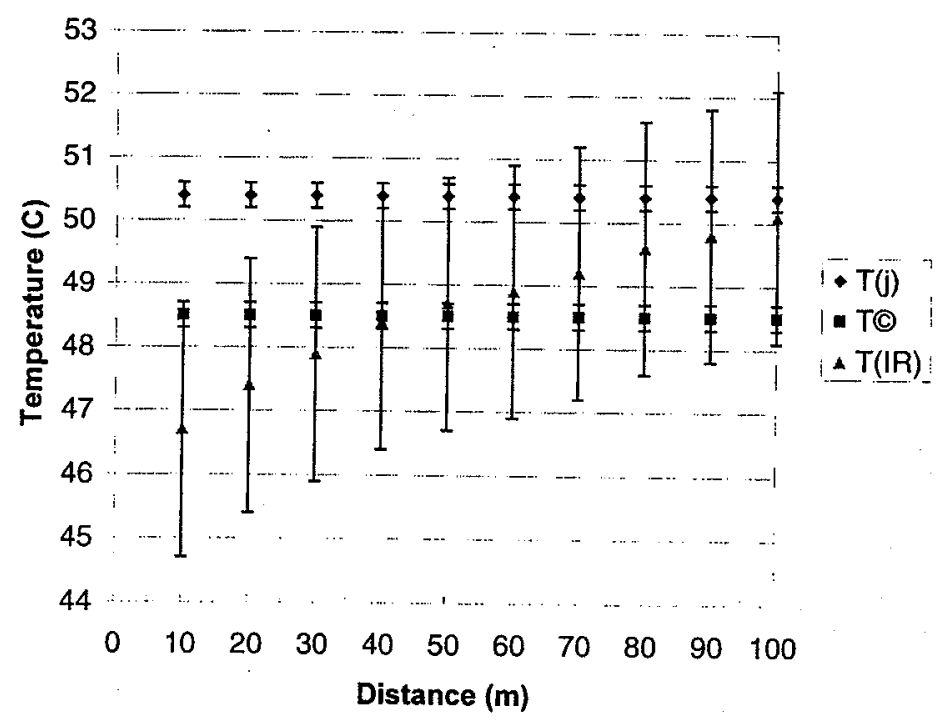

Fig. 6. Example of variation of the distance parametrs in built-in atmospheric model in order to compensate the effect of additional LDPE film attenuation 